\title{
The meaning of anti-Müllerian hormone levels in patients at a high risk of poor ovarian response
}

\author{
Hyun Jong Park', Geun Ho Lee', Du Sik Gong'1, Tae Ki Yoon², Woo Sik Lee ${ }^{2}$ \\ 'Department of Obstetrics and Gynecology, CHA Gumi Medical Center, Gumi; ${ }^{2}$ Department of Obstetrics and Gynecology, Fertility Center of CHA \\ Gangnam Medical Center, CHA University, Seoul, Korea
}

\begin{abstract}
Measurements of ovarian reserve play an important role in predicting the clinical results of assisted reproductive technology (ART). The ideal markers of ovarian reserve for clinical applications should have high specificity in order to determine genuine poor responders. Basal folliclestimulating hormone levels, antral follicle count, and serum anti-Müllerian hormone (AMH) levels have been suggested as ovarian reserve tests that may fulfill this requirement, with serum AMH levels being the most promising parameter. Serum AMH levels have been suggested to be a predictor of clinical pregnancy in ART for older women, who are at a high risk for decreased ovarian response. We reviewed the prognostic significance of ovarian reserve tests for patients undergoing ART treatment, with a particular focus on the significance of serum AMH levels in patients at a high risk of poor ovarian response.
\end{abstract}

Keywords: Anti-Müllerian hormone; Fertilization in vitro; Infertility; Pregnancy rate

\section{Introduction}

A decline in female fertility occurs after the age of 30 years and is accelerated after 35 years of age. It is known that women 40 years of age or older can generally be considered to be nearly infertile [1]. A woman's age can be a key factor in predicting the possibility of successful outcomes of in vitro fertilization. However, in actual clinical settings, even women of the same age can show considerable variability in fertility based on individual characteristics [2]. Roest et al. [3] reported that pregnancy outcomes after treatment with assisted reproductive technology (ART) among women 40 years of age or older were better in patients with a good response to controlled ovarian stimulation (COS) than in patients with a poor response. Thus, ovari-

Received: Mar 26, 2016 · Revised: Apr 16, 2016 · Accepted: May 18, 2016 Corresponding author: Woo Sik Lee

Department of Obstetrics and Gynecology, Fertility Center of CHA Gangnam Medical Center, CHA University, 566 Nonhyeon-ro, Gangnam-gu, Seoul 06135 , Korea

Tel: +82-2-3468-2840 Fax: +82-2-3468-2610 E-mail: wooslee@cha.ac.kr

This is an Open Access article distributed under the terms of the Creative Commons Attribution Non-Commercial License (http://creativecommons.org/licenses/by-nc/4.0/) which permits unrestricted non-commercial use, distribution, and reproduction in any medium, provided the original work is properly cited. an reserve tests predicting ovarian response to $\operatorname{COS}$ could potentially be used as indicators of the clinical outcomes of ART, such as pregnancy and live birth [4]. Eventually, screening patients using ovarian reserve tests to determine their prognosis for infertility treatment may play an important role in implementing individualized ART treatment. In this article, we reviewed the prognostic significance of ovarian reserve tests in the field of ART, with a particular focus on the significance of anti-Müllerian hormone (AMH) levels in patients at a high risk of poor ovarian response.

\section{Characteristics of valuable markers of ovarian reserve}

For in vitro fertilization-embryo transfer (IVF-ET) cycles, it has been established that four or more oocytes are generally needed to ensure the transfer of two embryos $[5,6]$. The Bologna criteria for defining poor responders classify cases in which three or fewer oocytes are retrieved in conventional COS for IVF-ET as poor responders [7]. Poor responders may be expected to have a relatively lower likelihood of clinical pregnancy. Accordingly, identifying ovarian reserve markers capable of diagnosing poor responders would be of major prognos- 
tic significance.

The 2015 American Society for Reproductive Medicine (ASRM) practice committee suggested that an ideal marker of ovarian reserve would be useful for predicting the possibility of pregnancy as well as for predicting the quantity and quality of oocytes [4]. Sensitivity and specificity are valuable indicators of the usefulness of diagnostic modalities. Given the general characteristics of ART, ovarian reserve markers with a high specificity would be ideal for reducing false positives and facilitating the differential diagnosis of genuine poor responders who require adoption or oocyte donation [4].

\section{Ovarian reserve markers}

Women experience several changes involving reproductive hormones as they grow older. A gradual decrease in the number of ovarian follicles with aging results in decreases in the levels of AMH and inhibin $B$, which are secreted from the primary, preantral, and antral follicles. Decreases in central negative feedback lead to a compensatory increase in the secretion of pituitary follicle-stimulating hormone (FSH). This is followed by an increase in late luteal and early follicular FSH levels. Thus, the earlier growth of new follicles is accelerated, which is accompanied by an increase in basal estradiol levels measured on days 2 to 3 of the menstrual cycle. The length of the follicular phase is shortened, meaning that the overall length of the menstrual cycle is also reduced. It has been hoped that indicators of changes in reproductive hormonal status during the aging process could be used as markers of ovarian reserve.

The ASRM practice committee reported that combining ovarian reserve markers to predict pregnancy may be clinically invalid due to the severe heterogeneity of the measurements, and that the predictive value of such a combination would not be greater than that of a single marker [4]. Therefore, we omitted any discussion of combinations of ovarian reserve markers.

\section{FSH}

Basal serum FSH levels have been shown to exhibit intense variability within the menstrual cycle or between cycles, which has limited their reliability as a marker of ovarian reserve [8-10]. However, in studies where FSH cut-off points of $\geq 10 \mathrm{IU} / \mathrm{L}$ were used, the specificity reached $83 \%$ to $100 \%$ in predicting poor response to COS (three or fewer follicles or four or fewer retrieved oocytes) [11], although FSH levels showed a lower sensitivity in predicting poor response. Scott et al. [12] reported that no live births occurred in patients with FSH levels exceeding $18 \mathrm{IU} / \mathrm{L}$. Thus, basal FSH levels may be useful as a measurement of ovarian reserve in diagnosing genuine poor responders.

However, it has been found that elevated basal FSH levels were not associated with an increase of aneuploidy in clinical pregnancy achieved using ART $[13,14]$. In addition, elevated FSH levels in women less than 40 years of age cannot be used as the sole indicator for predicting poor response and the possibility of clinical pregnancy during $\operatorname{COS}$ [15]. Overall, if clinicians predict ovarian reserve and pregnancy outcomes only based on basal FSH levels in women with a low risk of decreased ovarian response (DOR), false positives are likely.

\section{Estradiol and inhibin B}

Basal estradiol levels were found to show no significant differences between poor responders and normal ovarian responders [4]. Moreover, basal estradiol levels cannot be used as the sole criterion for screening poor responders. However, when infertile women had normal basal FSH levels with basal estradiol levels of $>60$ to $80 \mathrm{pg} /$ $\mathrm{mL}$ in the early follicular phase, limited evidence was found to support correlations with poor ovarian response, a higher cancellation rate of IVF-ET cycles, and lower rates of pregnancy during ART [1618]. Therefore, basal estradiol levels may be of minor significance as a supplemental measure in hormonal assessments of women with normal basal FSH levels [4].

Inhibin B was found to show intense intracycle variability, and therefore cannot be used as a reliable marker [4].

\section{Antral follicle count}

The antral follicle count (AFC) exhibits sufficient intercycle reliability and interobserver reliability for measuring ovarian reserve. In 2005, Hendriks et al. [19] performed a meta-analysis and reported that an AFC below the average of 5.2 (range, 3-6) was associated with poor responders, but the AFC did not have a prognostic value for pregnancy failure. In fact, if the presence of three to four follicles was used as a cut-off value for low AFC, higher levels of specificity (73\%-100\%) would have been achieved in predicting poor ovarian response. Moreover, the AFC also had a specificity of $64 \%-100 \%$ in predicting pregnancy failure [4]. Therefore, the AFC could potentially be used as an indicator to predict genuine cases of poor ovarian response. However, it has been found that the sensitivity of AFC was relatively low in predicting poor ovarian response and pregnancy failure [19].

\section{4. $\mathrm{AMH}$}

AMH is directly secreted from the preantral and antral follicles, and has the useful property of reflecting the age-induced decrease in the number of follicles. Serum AMH levels are known to be a highly reliable marker for measuring ovarian reserve because they are not affected by gonadotropin, and AMH exhibits minimal variability within or among menstrual cycles. Currently, the presence of a proportional relationship between AMH levels and the ovarian response to $\mathrm{COS}$ is 
commonly accepted [4]. According to recent studies, serum AMH levels have been found to better reflect ovarian reserve than age or basal levels of FSH, estradiol, and inhibin B [20].

\section{1) Studies of AMH levels in populations at a low risk for DOR}

According to Hazout et al. [21], serum AMH levels showed a significant positive correlation with the number of metaphase II oocytes retrieved during COS-IVF cycles $(r=0.38, p<0.05)$. Moreover, the number of embryos that were obtained also had a significant positive correlation with serum AMH levels $(r=0.34, p<0.05)$. In studies assessing embryo quality, Smeenk et al. [22] and Takahashi et al. [23] reported no significant correlations between serum AMH levels and embryo quality. Ebner et al. [24] reported that the highest-quality oocytes were obtained from patients whose serum AMH levels ranged between $1.66 \mathrm{ng} / \mathrm{mL}$ and $4.52 \mathrm{ng} / \mathrm{mL}$, but that no significant difference was noted in the acquisition of high-quality embryos among patient groups sorted by AMH levels. Silberstein et al. [25] found that the area under the curve (AUC) of serum AMH levels was 0.647 (poor) in predicting the acquisition of high-quality embryos, which was argued to reflect the poor clinical applicability of AMH levels.

Regarding the clinical pregnancy rate, Gnoth et al. [26] ( $n=132)$ reported no significant difference between patients with high levels of $\mathrm{AMH}$ and patients with low levels of AMH based on a cut-off value of $1.26 \mathrm{ng} / \mathrm{mL}$. Silberstein et al. [25] $(\mathrm{n}=257)$ observed a significant difference in the rate of implantation ( $28.0 \%$ vs. $16.8 \%, p<0.001)$, but no significant difference in the ongoing pregnancy rate $(46.4 \%$ vs. $34.9 \%, p=0.084$ ) between high-AMH and low-AMH groups, defined using a cut-off value of $2.7 \mathrm{ng} / \mathrm{mL}$. In contrast, Elgindy et al. [27] reported that an AMH cut-off value of $2.7 \mathrm{ng} / \mathrm{mL}$ showed a specificity of $82.4 \%$ in predicting clinical pregnancy, and therefore suggested that it could be useful as a clinical predictor; but their study had the limitation of only including 33 patients. Eldar-Geva et al. [28] reported that serum AMH levels were a useful predictor of clinical pregnancy using an AUC value of 0.75 (fair); but that study similarly had the limitation of only enrolling 56 patients. Therefore, the results of those studies cannot be generalized. A meta-analysis of individual patient data published in 2013 (including 28 studies, $n=5,705$ ) suggested that the AUC of serum AMH levels was 0.55 (failure) for the prediction of clinical pregnancy [29]. According to a recent meta-analysis published in 2015 (including 19 studies, $n=5,373$ ), serum AMH levels had an AUC of 0.634 (poor) for the prediction of clinical pregnancy [30]. These results collectively suggest that serum AMH levels cannot be used as a predictor of clinical pregnancy in patients with normal ovarian response.

With regard to the prediction of live births, Nelson et al. [31] and Lukaszuk et al. [32] reported AUC values of serum AMH levels of 0.62

ํํำ 
(poor) and 0.61 (poor), respectively, suggesting that AMH levels cannot be applied in clinical settings for this purpose. In addition, according to a meta-analysis including 13 studies that was published in 2014, serum AMH levels showed an AUC of 0.61 (poor) in predicting live births, suggesting poor applicability in the clinical setting [33].

In summary, serum AMH levels cannot be used to predict the clinical outcomes of ART in patients with normal ovarian response (Table 1).

\section{2) Studies of AMH levels in populations at a high risk for DOR}

In a study focusing on the cancellation rate of IVF cycles, Penarrubia et al. [34] reported a specificity of $96 \%$ for the AMH cut-off value of $0.7 \mathrm{ng} / \mathrm{mL}$, thus suggesting that AMH levels exhibited promise as a predictive indicator. Muttukrishna et al. [35] conducted a prospective study in women with basal FSH levels $>10 \mathrm{IU} / \mathrm{L}$ or aged $>38$ years, and reported significant differences in the serum AMH levels of patients whose IVF cycles were cancelled due to poor ovarian response in comparison to the normal control group $(0.175 \pm 0.04 \mathrm{ng} / \mathrm{mL}$ vs. $1.13 \pm 0.2 \mathrm{ng} / \mathrm{mL}, p<0.05$ ). Mcllveen et al. [36] reported that the AUC value of serum AMH levels was 0.78 (fair) in predicting the cancellation of IVF cycles in poor responders, thereby validating serum AMH levels as an indicator predictive of the cancellation of IVF cycles. Furthermore, they reported a sensitivity of $85 \%$ and a specificity of $63 \%$ for the cancellation of IVF cycles using an AMH cut-off value of 1.25 $\mathrm{ng} / \mathrm{mL}$. Kim et al. [37] performed a retrospective analysis of 176 IVF/ intracytoplasmic sperm injection cycles and showed a sensitivity of $38 \%$ and a specificity of $95 \%$ in predicting DOR at an $\mathrm{AMH}$ level of $\leq 0.76 \mathrm{ng} / \mathrm{mL}$, with DOR defined as two or fewer total retrieved oocytes or one or fewer mature oocytes. They also reported that the AUC of serum AMH levels in predicting DOR was 0.787 (fair), which was a higher value than the AUC values of FSH (0.712) and age (0.730)

In a study evaluating embryo quality, Kavoussi et al. [38] reported that regardless of age, low serum AMH levels ( $<1 \mathrm{ng} / \mathrm{mL}$ ) were associated with a significantly lower availability of high-quality blastocysts for cryopreservation than was observed in patients with higher AMH levels (1-4 ng/mL) in IVF cycles.

Regarding clinical pregnancy rates, Mcllveen et al. [36] reported that serum AMH levels could not be used as a predictive factor for clinical pregnancy during ART in women aged $\geq 39$ years or in poor ovarian responders. They pointed out that serum AMH levels are associated with the measurement of oocyte quantity rather than oocyte quality. In contrast, in a large-scale retrospective study on 1,558 patients, Wang et al. [39] found a significant positive correlation between serum AMH levels and the rate of clinical pregnancy in women aged between 34 and 41 years. The researchers also noted that in women $\geq 42$ years of age, the rate of clinical pregnancy was markedly lower (3\%) in patients with serum AMH levels $\leq 0.29 \mathrm{ng} / \mathrm{mL}$, in comparison to $\geq 17 \%$ in patients with serum AMH levels exceeding $0.3 \mathrm{ng} / \mathrm{mL}$. According to a retrospective study conducted by Sahmay et al. [40], serum AMH levels had an AUC of 0.790 (fair) in predicting clinical pregnancy in 240 women aged $\geq 35$ years. It was suggested that serum $\mathrm{AMH}$ levels could be a reliable indicator for predicting clinical pregnancy, as an AMH cut-off value of $1.91 \mathrm{ng} / \mathrm{mL}$ showed a sensitivity of $74 \%$ and a specificity of $73 \%$ in predicting clinical pregnancy. Gomez et al. [41] reported that serum AMH levels decreased by approximately $0.384 \mathrm{ng} / \mathrm{mL}$ every year in a large-scale retrospective study of 1,287 patients, which was found to be a significant decrease. In women aged $<36$ years, serum AMH levels did not affect the rate of clinical pregnancy. However, in women aged $\geq 36$ years, a gradual age-related increase was observed in the degree of correlation between the clinical pregnancy rate and serum AMH levels. According to the retrospective study of Park et al. [42] of 188 Korean women aged $\geq 40$ years, serum AMH levels had an AUC of 0.721 (fair) in predicting clinical pregnancy. At an AMH cut-off value of $1.90 \mathrm{ng} /$ $\mathrm{mL}$, the rate of clinical pregnancy was 6.731-fold higher in the highAMH group than in the low-AMH group (19/54 [35.2\%] vs. 10/134 [7.5\%], $p<0.001)$. In contrast, Friden et al. [43] conducted a retrospective study in women aged $\geq 39$ years, and found that serum AMH levels had an AUC of 0.65 (poor) in predicting pregnancy failure during $A R T$, thus suggesting that serum $A M H$ values have relatively poor clinical applicability. According to a recent meta-analysis published in 2015, serum AMH levels had an overall summarized AUC of 0.696 (95\% confidence interval [Cl], 0.641-0.751) (close to fair), an overall summarized sensitivity of $69.9 \%$ (95\% Cl, 61.0\%-77.9\%), and an overall summarized specificity of $64.7 \%(95 \% \mathrm{Cl}, 60.9 \%-68.3 \%)$ in predicting clinical pregnancy in poor responders $(n=615)$, indicating that serum AMH levels may be a weak predictor of clinical pregnancy in poor responders [30] (Table 2).

\section{Conclusions}

Summarizing the literature reviewed above, three indicators ( $F S H$, $\mathrm{AFC}$, and $\mathrm{AMH}$ ) have showed utility in assessing ovarian reserve, and among these indicators, serum AMH levels are the most promising parameter. However, serum AMH levels may be a diagnostic test for poor ovarian response rather than a screening test for ovarian reserve $[44,45]$. The significance of serum AMH levels in predicting clinical pregnancy during ART treatment is lower in patients with a low risk of DOR. The predictive value of serum AMH levels for clinical pregnancy seems to gradually increase in older women with an increased risk of poor ovarian response. These findings may be explained by the following mechanism. The proportion of good-quality oocytes is reduced with the decreased pool of oocytes in older women. However, women of the same age with higher ovarian reserve 


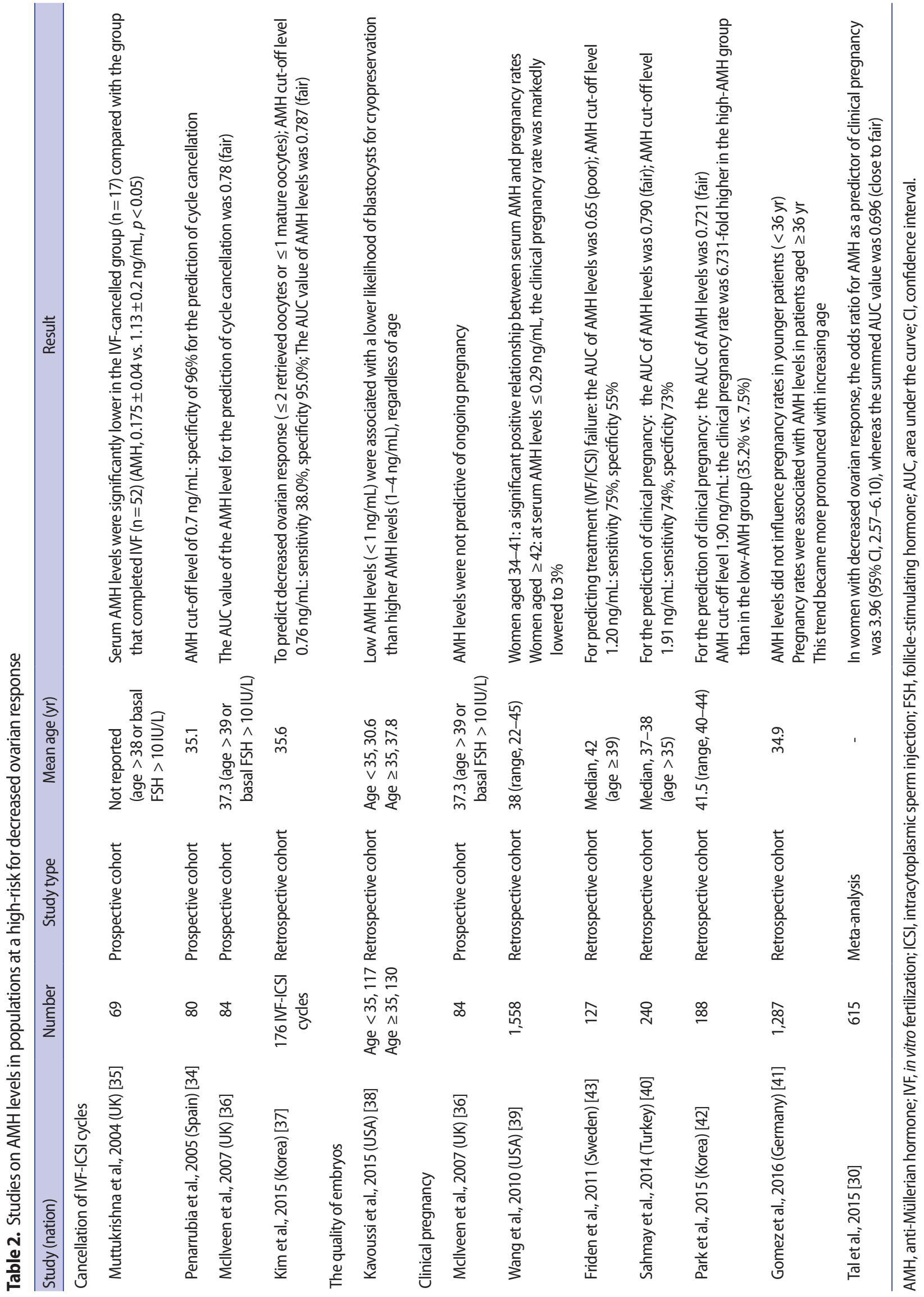


(indicated by elevated serum AMH levels), which could lead to a quantitative compensation for the age-related decrease in the quality of oocytes, may show better pregnancy outcomes in ART treatment compared to other women with lower ovarian reserve [4]. Further large-scale prospective studies are needed to determine the optimal cut-off level of serum AMH for predicting the prognosis of ART in patients at a high risk of poor ovarian response.

\section{Conflict of interest}

No potential conflict of interest relevant to this article was reported.

\section{References}

1. Spira A. The decline of fecundity with age. Maturitas 1988;Suppl 1:15-22.

2. te Velde ER, Pearson PL. The variability of female reproductive ageing. Hum Reprod Update 2002;8:141-54.

3. Roest J, van Heusden AM, Mous H, Zeilmaker GH, Verhoeff A. The ovarian response as a predictor for successful in vitro fertilization treatment after the age of 40 years. Fertil Steril 1996;66: 969-73.

4. Practice Committee of the American Society for Reproductive Medicine. Testing and interpreting measures of ovarian reserve: a committee opinion. Fertil Steril 2015;103:e9-e17.

5. Bancsi LF, Broekmans FJ, Eijkemans MJ, de Jong FH, Habbema $J D$, te Velde ER. Predictors of poor ovarian response in in vitro fertilization: a prospective study comparing basal markers of ovarian reserve. Fertil Steril 2002;77:328-36.

6. Klinkert ER, Broekmans FJ, Looman CW, Te Velde ER. A poor response in the first in vitro fertilization cycle is not necessarily related to a poor prognosis in subsequent cycles. Fertil Steril 2004; 81:1247-53.

7. Ferraretti AP, La Marca A, Fauser BC, Tarlatzis B, Nargund G, Gianaroli $L$, et al. ESHRE consensus on the definition of 'poor response' to ovarian stimulation for in vitro fertilization: the Bologna criteria. Hum Reprod 2011;26:1616-24.

8. Jayaprakasan K, Campbell B, Hopkisson J, Clewes J, Johnson I, Raine-Fenning N. Establishing the intercycle variability of threedimensional ultrasonographic predictors of ovarian reserve. Fertil Steril 2008;90:2126-32.

9. Scott RT Jr, Hofmann GE, Oehninger S, Muasher SJ. Intercycle variability of day 3 follicle-stimulating hormone levels and its effect on stimulation quality in in vitro fertilization. Fertil Steril 1990;54:297-302.

10. Kwee J, Schats R, McDonnell J, Lambalk CB, Schoemaker J. Intercycle variability of ovarian reserve tests: results of a prospective randomized study. Hum Reprod 2004;19:590-5.

11. Esposito MA, Coutifaris C, Barnhart KT. A moderately elevated day $3 \mathrm{FSH}$ concentration has limited predictive value, especially in younger women. Hum Reprod 2002;17:118-23.

12. Scott RT Jr, Elkind-Hirsch KE, Styne-Gross A, Miller KA, Frattarelli $\mathrm{JL}$. The predictive value for in vitro fertility delivery rates is greatly impacted by the method used to select the threshold between normal and elevated basal follicle-stimulating hormone. Fertil Steril 2008;89:868-78.

13. Thum MY, Abdalla HI, Taylor D. Relationship between women's age and basal follicle-stimulating hormone levels with aneuploidy risk in in vitro fertilization treatment. Fertil Steril 2008;90: 315-21.

14. Massie JA, Burney RO, Milki AA, Westphal LM, Lathi RB. Basal follicle-stimulating hormone as a predictor of fetal aneuploidy. Fertil Steril 2008;90:2351-5.

15. Roberts JE, Spandorfer S, Fasouliotis SJ, Kashyap S, Rosenwaks Z. Taking a basal follicle-stimulating hormone history is essential before initiating in vitro fertilization. Fertil Steril 2005;83:37-41.

16. Evers JL, Slaats P, Land JA, Dumoulin JC, Dunselman GA. Elevated levels of basal estradiol-17beta predict poor response in patients with normal basal levels of follicle-stimulating hormone undergoing in vitro fertilization. Fertil Steril 1998;69:1010-4.

17. Licciardi FL, Liu HC, Rosenwaks Z. Day 3 estradiol serum concentrations as prognosticators of ovarian stimulation response and pregnancy outcome in patients undergoing in vitro fertilization. Fertil Steril 1995;64:991-4.

18. Smotrich DB, Widra EA, Gindoff PR, Levy MJ, Hall JL, Stillman RJ. Prognostic value of day 3 estradiol on in vitro fertilization outcome. Fertil Steril 1995;64:1136-40.

19. Hendriks DJ, Mol BW, Bancsi LF, Te Velde ER, Broekmans FJ. Antral follicle count in the prediction of poor ovarian response and pregnancy after in vitro fertilization: a meta-analysis and comparison with basal follicle-stimulating hormone level. Fertil Steril 2005;83:291-301.

20. La Marca A, Sighinolfi G, Radi D, Argento C, Baraldi E, Artenisio $\mathrm{AC}$, et al. Anti-Mullerian hormone (AMH) as a predictive marker in assisted reproductive technology (ART). Hum Reprod Update 2010;16:113-30.

21. Hazout A, Bouchard P, Seifer DB, Aussage P, Junca AM, CohenBacrie P. Serum antimullerian hormone/mullerian-inhibiting substance appears to be a more discriminatory marker of assisted reproductive technology outcome than follicle-stimulating hormone, inhibin B, or estradiol. Fertil Steril 2004;82:1323-9.

22. Smeenk JM, Sweep FC, Zielhuis GA, Kremer JA, Thomas CM, Braat $D D$. Antimullerian hormone predicts ovarian responsiveness, but not embryo quality or pregnancy, after in vitro fertilization or in- 
tracyoplasmic sperm injection. Fertil Steril 2007;87:223-6.

23. Takahashi C, Fujito A, Kazuka M, Sugiyama R, Ito H, Isaka K. AntiMullerian hormone substance from follicular fluid is positively associated with success in oocyte fertilization during in vitro fertilization. Fertil Steril 2008;89:586-91.

24. Ebner T, Sommergruber M, Moser M, Shebl O, Schreier-Lechner E, Tews $G$. Basal level of anti-Mullerian hormone is associated with oocyte quality in stimulated cycles. Hum Reprod 2006;21:20226.

25. Silberstein T, MacLaughlin DT, Shai I, Trimarchi JR, Lambert-Messerlian G, Seifer DB, et al. Mullerian inhibiting substance levels at the time of HCG administration in IVF cycles predict both ovarian reserve and embryo morphology. Hum Reprod 2006;21:15963.

26. Gnoth C, Schuring AN, Friol K, Tigges J, Mallmann P, Godehardt E. Relevance of anti-Mullerian hormone measurement in a routine IVF program. Hum Reprod 2008;23:1359-65.

27. Elgindy EA, El-Haieg DO, El-Sebaey A. Anti-Mullerian hormone: correlation of early follicular, ovulatory and midluteal levels with ovarian response and cycle outcome in intracytoplasmic sperm injection patients. Fertil Steril 2008;89:1670-6.

28. Eldar-Geva T, Ben-Chetrit A, Spitz IM, Rabinowitz R, Markowitz E, Mimoni T, et al. Dynamic assays of inhibin $B$, anti-Mullerian hormone and estradiol following FSH stimulation and ovarian ultrasonography as predictors of IVF outcome. Hum Reprod 2005;20: 3178-83.

29. Broer SL, van Disseldorp J, Broeze KA, Dolleman M, Opmeer BC, Bossuyt $\mathrm{P}$, et al. Added value of ovarian reserve testing on patient characteristics in the prediction of ovarian response and ongoing pregnancy: an individual patient data approach. Hum Reprod Update 2013;19:26-36.

30. Tal R, Tal O, Seifer BJ, Seifer DB. Antimullerian hormone as predictor of implantation and clinical pregnancy after assisted conception: a systematic review and meta-analysis. Fertil Steril 2015;103: 119-30.e3.

31. Nelson SM, Yates RW, Fleming R. Serum anti-Mullerian hormone and FSH: prediction of live birth and extremes of response in stimulated cycles. Implications for individualization of therapy. Hum Reprod 2007;22:2414-21.

32. Lukaszuk K, Liss J, Kunicki M, Jakiel G, Wasniewski T, WoclawekPotocka I, et al. Anti-Mullerian hormone (AMH) is a strong predictor of live birth in women undergoing assisted reproductive technology. Reprod Biol 2014;14:176-81.

33. Iliodromiti S, Kelsey TW, Wu O, Anderson RA, Nelson SM. The predictive accuracy of anti-Mullerian hormone for live birth after assisted conception: a systematic review and meta-analysis of the literature. Hum Reprod Update 2014;20:560-70.

34. Penarrubia J, Fabregues F, Manau D, Creus M, Casals G, Casamitjana $R$, et al. Basal and stimulation day 5 anti-Mullerian hormone serum concentrations as predictors of ovarian response and pregnancy in assisted reproductive technology cycles stimulated with gonadotropin-releasing hormone agonist-gonadotropin treatment. Hum Reprod 2005;20:915-22.

35. Muttukrishna S, Suharjono H, McGarrigle H, Sathanandan M. Inhibin $B$ and anti-Mullerian hormone: markers of ovarian response in IVF/ICSI patients? BJOG 2004;111:1248-53.

36. Mcllveen M, Skull JD, Ledger WL. Evaluation of the utility of multiple endocrine and ultrasound measures of ovarian reserve in the prediction of cycle cancellation in a high-risk IVF population. Hum Reprod 2007;22:778-85.

37. Kim SK, Lee JR, Jee BC, Suh CS, Kim SH. What number of oocytes is appropriate for defining poor ovarian response? Yonsei Med J 2015;56:482-9.

38. Kavoussi SK, Odenwald KC, Boehnlein LM, Summers-Colquitt RB, Pool TB, Swain JE, et al. Antimullerian hormone as a predictor of good-quality supernumerary blastocyst cryopreservation among women with levels $<1 \mathrm{ng} / \mathrm{mL}$ versus $1-4 \mathrm{ng} / \mathrm{mL}$. Fertil Steril 2015;104:633-6.

39. Wang JG, Douglas NC, Nakhuda GS, Choi JM, Park SJ, Thornton $\mathrm{MH}$, et al. The association between anti-Mullerian hormone and IVF pregnancy outcomes is influenced by age. Reprod Biomed Online 2010;21:757-61.

40. Sahmay S, Oncul M, Tuten A, Tok A, Acikgoz AS, Cepni I. Antimullerian hormone levels as a predictor of the pregnancy rate in women of advanced reproductive age. J Assist Reprod Genet 2014;31:1469-74.

41. Gomez R, Schorsch M, Hahn T, Henke A, Hoffmann I, Seufert R, et al. The influence of AMH on IVF success. Arch Gynecol Obstet 2016;293:667-73.

42. Park HJ, Lyu SW, Seok HH, Yoon TK, Lee WS. Anti-Mullerian hormone levels as a predictor of clinical pregnancy in in vitro fertilization/intracytoplasmic sperm injection-embryo transfer cycles in patients over 40 years of age. Clin Exp Reprod Med 2015;42: 143-8.

43. Friden B, Sjoblom P, Menezes J. Using anti-Mullerian hormone to identify a good prognosis group in women of advanced reproductive age. Aust N Z J Obstet Gynaecol 2011;51:411-5.

44. Maheshwari A, Gibreel A, Bhattacharya S. Screening for early ovarian ageing. In: Bewley S, Ledger W, Nikolaou D, editors. Reproductive ageing. London: RCOG Press; 2009. p.201-10.

45. Loh JS, Maheshwari A. Anti-Mullerian hormone: is it a crystal ball for predicting ovarian ageing? Hum Reprod 2011;26:2925-32. 